\title{
Lipid rafts interaction of the ARID3A transcription factor with EZRIN and G-Actin regulates B cell receptor signaling.
}

Christian Schmidt ${ }^{1,2}$, Laura Christian ${ }^{1,3}$, Josephine Tidwell ${ }^{1}$, Dongkyoon Kim ${ }^{1,4}$ and Haley O Tucker ${ }^{1,5}$

${ }^{1}$ Department of Molecular Biosciences, the University of Texas at Austin, Austin TX, 78712. USA.

${ }^{2}$ Current Address: Department of Biomaterials and Healthcare, Division of Life Science and

Bioprocesses, Fraunhofer-Institute for Applied Polymer Research (IAP), 14476 Potsdam-Golm, Germany.

${ }^{3}$ Current Address: Department of Biological Sciences, Rensselaer Polytechnic Institute; Troy, New York, 12180, USA.

${ }^{4}$ Current Address: Atreca, Inc., South San Francisco, California 94080, USA.

${ }^{5}$ Corresponding author: Haley O. Tucker, Professor and Morrow Centennial Chair; Molecular Biosciences, Institute for Cellular and Molecular Biology, University of Texas at Austin, 1 University

Station A5000, Austin TX 78712. haleytucker@austin.utexas.edu

Running Title: ARID3A interacts with EZRIN to regulate BCR signaling.

KEY WORDS transcriptional regulation, lipid rafts, Actin cytoskeleton, B cell antigen receptor

\section{ABSTRACT}

Previously we demonstrated that the ARID3A transcription factor shuttles between the nucleus and the plasma membrane, where it localizes within lipid rafts. There it interacts with components of the $B$ Cell Receptor $(B C R)$ to reduce its ability to transmit downstream signaling. We demonstrate in this report that a direct component of ARID3A-regulated BCR signal strength is cortical Actin. ARID3A interacts with Actin exclusively within lipid rafts via the Actin binding protein EZRIN, which confines unstimulated BCRs within lipid rafts. BCR ligation discharges the ARID3A-EZRIN complex from lipid rafts allowing the BCR to initiate downstream signaling events. The ARID3A-EZRIN interaction occurs almost exclusively within unpolymerized G-Actin where EZRIN interacts with the multifunctional ARID3A REKLES domain. These observations provide a novel mechanism by which a transcription factor directly regulates $B C R$ signaling. 


\section{INTRODUCTION}

The cortical cytoskeleton is an Actin-based structure that underlies and confers mechanical support to the plasma membrane (PM) via a dense meshwork ( 100 nm thick) comprised primarily of filamentous (F) Actin (1). An array of proteins, including myosin motors as well as Actin-binding and linker factors comprise the PM (2). The cortex is dynamic in that it can locally disassemble (G-Actin) and reassemble (F-Actin) to drive formation of cellular protrusions and to modulate lipid microdomain (lipid raft) organization $(3,4)$. PM-associated receptors maintain dynamic contacts with the cortex via linker proteins that simultaneously bind Actin and the cytoplasmic domains of PM proteins (5). The resulting receptor-cytoskeleton anchoring has a direct impact on receptor mobility and influences receptor function (6). Over 100 Actin-binding proteins constitute the cortical cytoskeleton (1).

In resting $B$ cells, the mobility of $B$ Cell Antigen Receptors (BCRs) is restricted, as the cortical Actin cytoskeleton acts as a barrier to their diffusion $(7,8)$. Such contractile stress and tension is mediated by ERM proteins (EZZRIN, $\underline{R A D I X I N ~ a n d ~} \underline{M O E S I N)}(9,10)$. ERM proteins supply the tension needed to anchor B Cell Receptors (BCRs) in the cortex. Actin and EZRIN form a network which confines BCRs in nanoscale lipid rafts via EZRIN carboxyl terminal binding to integral membrane proteins of the BCR complex (11). BCR mobility, on the other hand, is established if the EZRIN-Actin interaction is disrupted (12).

Besides its mechanical functions, Actin has also been implicated in transcriptional regulationboth through cytoplasmic alterations in cytoskeletal ACTIN dynamics and through the assembly of transcription factor (TF) regulatory complexes (13). Established examples include the subcellular localization of myocardin-related TFs (MAL, MKL1, BSAC and MRTF-B) (14, 15), the PREP2 homeoprotein TF, and the TF repressor, YY1 $(16,17)$.

We demonstrated (18) that a palmitoylated pool of ARID3A, a B-cell/embryonic stem cellrestricted $T F$, shuttles from the nucleus to the PM where it is diverted to lipid rafts of resting $B$ cells to associate with "signalosome" components. The BCR signalosome requires participation of signaling proteins (eg, BTK and BLNK) whose genetic defects often impair B cell activation, differentiation and often lead to agammaglobulinemia $(11,12)$. Following BCR stimulation, ARID3A transiently interacts with SUMOylation enzymes, blocks calcium flux and phosphorylation of BTK and TFII-I TFs and is then discharged from lipid rafts as a SUMO-I-modified form (18). The lipid raft concentration of ARID3A contributes to the signaling threshold of $B$ cells, as their sensitivity to BCR stimulation decreases as the 
levels of ARID3A increase (18). These events are independent of the previously established role of nuclear ARID3A in immunoglobulin gene transcription $(19,20)$.

We concluded from the results above that ARID3A contributes to a BCR tuning mechanism within lipid rafts, yet further details of how this mechanism is initiated at the PM remained unknown. Here we demonstrate that a critical component underlying ARID3A-regulated BCR signal strength is Actin. ARID3A interacts with Actin, exclusively within lipid rafts, via the Actin-binding protein, EZRIN, which in unstimulated BCRs is confined within lipid rafts. We observed that antigen binding discharges the ARID3A-EZRIN complex from lipid rafts allowing initiation of downstream signaling events. Consistent with the timing of EZRIN discharge, ARID3A-EZRIN interaction occurs almost exclusively within unpolymerized, G-Actin, and is mediated by the multifunctional REKLES domain of ARID3A. These along with our previous data provide further mechanistic insight as to how TFs can directly regulate BCR signaling strength.

\section{RESULTS and DISCUSSION}

Variable levels of ARID3A within lipid raft in unstimulated and stimulated $B$ cell lymphomas. From B cell leukemias/lymphomas, we prepared whole cell lysates (WCL) as previously described (18). Lipid rafts were prepared by flotation on discontinuous sucrose gradients (18) using the B cell-specific lipid rafts component, Raflin (21), as an internal control for purity (Materials and Methods). Plasma membranes (PM), isolated as pellets from the same raft extraction, were further purified for 30 min on ice as described in Materials and Methods.

As shown in S-Figure 1, we estimated that raft-localized ARID3A accounted for $<10 \%$ of total cellular ARID3A - a value similar to the previously estimated level of membrane $(\mathrm{m})$ lgM in lipid rafts (22, 23). Lipid rafts from Raji and Daudi Burkett lymphomas contained 10-fold less ARID3A than those of the CL01 B cell leukemia or the Ramos Burkett lymphoma (S-Fig. 1). Equal loading was demonstrated by probing the blots with anti-Raftlin. No significant differences were observed in either WCLs or M fractions. The differences among these lines in raft-localized ARID3A made them excellent choices for our analyses of anti-BCR signal strength.

BCR stimulation induces a rafts-restricted, transient interaction of ARID3A with EZRIN prior to their co-discharge. We previously demonstrated that Ramos and CL01 were less sensitive, whereas Raji and Daudi were more sensitive to BCR stimulation (18). We further showed that engagement of the BCR results in a significant and specific reduction of the small pool of lipid raftslocalized ARID3A, which is lost from lipid rafts, as BTK and other signalosome components accumulate there in proportion to BCR signaling strength (18). 
That the cytoskeletal linker protein EZRIN undergoes a similar anti-IgM-mediated discharge from lipid rafts $(11,12)$ prompted us to examine ARID3A-EZRIN interaction under differential BCR signal strength conditions. We observed that EZRIN and ARID3A did not form an immunoprecipitable complex in WCL of any of the B cell lines, regardless if the cells were stimulated or not [Fig. $1 \mathrm{~A}(\mathrm{a}-\mathrm{C})$, lanes 5,6 , 11, 12, 17, 18, 23 and 24]. However, EZRIN and ARID3A did associate within lipid rafts, and their interaction required BCR stimulation (compare lanes 7 and 19 with lanes 8 and 20 in Fig. $1 A(a, b)$.

EZRIN was co-discharged with ARID3A as evidenced by their identical trafficking. That is, EZRIN was detected in anti-ARID3A IPs of lipid rafts of the higher threshold cell lines (Ramos and CL-01) stimulated with anti-IgM only [Fig. 1A(a), lanes 8 and 20); within all cell lines stimulated with anti-CD19 only [Fig. 1A(b), lanes 2, 8, 14 and 20]; and discharged in all cell lines following anti-lgM + anti-CD19 [Fig. 1A(c), lanes 2, 8, 14 and 20] stimulation. Notably, EZRIN was retained in the membrane fraction only following strong co-stimulation [Fig. 1A(c), Lanes 4, 10, 16 and 22]. We observed no change in the expression levels of SUMO-1, which modifies ARID3A at a single residue and interacts with ARID3A in rafts (18; S-Fig. 2).

These results suggested that post-discharge, the ARID-EZRIN-complex is directed to different fates depending on the signaling threshold of the BCR.

EZRIN knockdown results in reduced BCR signaling strength. The above results suggested that, within lipid rafts, EZRIN might link ARID3A indirectly to the BCR to control signaling strength. Thus, we tested if signal strength was impaired following (anti-lgM + anti-CD19) stimulation in B cells deficient in EZRIN.

Retroviral shRNA knockdown (KD) of EZRIN was assessed and validated in S-Figure 3 as $7590 \%$ relative to a scrambled sh-RNA KD control. EZRIN WT and KD B cell leukemias/lymphomas were loaded with Indo-1, incubated with an Fcy III/II receptor-blocking antibody (to prevent antibody-antigen immune complexes), and then strongly stimulated with anti-CD19 + F(ab')2-lgM 30 seconds (s) prior to measurement of $\mathrm{Ca}^{2+}$ flux. Cells were gated and representative results of three separate experiments are shown in Figure 1B. EZRIN loss led to significant reduction in signaling strength of the more sensitive Raji and Daudi B cells as compared to the less sensitive Ramos and CL01 B cells. That is, 38\% of Raji and Daudi responded to strong BCR stimulation as compared to $\sim 12 \%$ of Ramos and CL01.

These data lead us to speculate that the ARID3A-EZRIN complex might function to secure the raft environment for the active BCR complex or to prepare inactive BCR complexes for signaling activity. They further implied that the inherent signaling strength of the BCR, in the absence of EZRIN, may 
contribute in a feedback manner by directing the ultimate fates of ARID3A-EZRIN complexes within the Actin cytoskeleton-a hypothesis tested below.

ARID3A interacts via EZRIN with Actin. Previous reports established that EZRIN binds specifically to polymerized F-Actin $(11,12)$ and that partial depolymerization of Actin increased the strength of BCR signaling (24). This and the data above suggested that EZRIN mediates BCR ligationdependent aggregation of lipid rafts by releasing them from the underlying cortical Actin cytoskeleton.

As shown in Figure 2A, Actin and ARID3A interact in lipid rafts of all unstimulated $B$ cell lines (lanes 1, 5, 9 and 13). Strong stimulation with anti-lgM + anti CD19 (Lanes 4, 8, 12, and 16) discharged ARID3A from Actin in lipid rafts of all cells. Weaker stimulation with either anti-CD19 or anti-IgM discharged Actin from ARID3A in lipid rafts of strong signaling strength B cells (Raji and Daudi; Lanes 2, 3, 14 and 15), but not from weak signaling strength B cells (Ramos and CL-01; Lanes 6, 7, 10 and 11). Strong stimulation (Fig. 2A; lanes 4, 6, 12 and 16) was required to discharge from lipid rafts other ARID3A interacting proteins, including UBC-9, PIAS-1 and SUMO-I.

That ARIA3A was detected in lipid rafts implied it was discharged after strong stimulation. However, our data do not exclude that other, unknown proteins also were discharged, as no antibodies against these putative factors were employed.

Support for an ARID3A-Actin interaction was provided by computerized 3D reconstruction of immunofluorescence data. As shown in Figure 2B, reconstructions (detailed in Materials and Methods) of strongly stimulated murine B cells stained for Actin (green), ARID3A (red) and nuclei (blue) revealed strong Actin-ARID3A association (yellow; also illustrated in the movies of S-Figure 4)

ARID3A localizes within monomeric G-Actin. We reasoned that a functional consequence of ARID3A discharge is to release lipid rafts-associated Actin either for depolymerization or for polymerization. The degree to which release is achieved might contribute to the signaling threshold of the BCR.

In nonmuscle cells of mammals, Actin is encoded by two genes: Actb and Actg1, which encode beta-Actin and gamma-Actin respectively. Each isoform can exist as a monomer, termed Globular (G)Actin, or as a polymer, Filamentous (F)-Actin $(25,26)$. Changes in Actin organization are driven by the assembly and disassembly of F-Actin. This dynamic turnover is regulated by a diverse set of proteins, many of which bind to F-Actin to influence the network architecture $(27,28)$.

Following transfection of ARID3A into COS7 cells, we employed Triton X-100 with or without TWEEN 20 fractionation to obtain detergent-soluble (enriched in G-Actin) and insoluble (enriched in FActin) proteins as described in Materials and Methods. Following lysate fractionation over SDS-PAGE, 
Western blotting was performed to determine the partitioning of ARID3A and EZRIN. Acid sphingomyelinase (SMPD1) served as a positive control for the insoluble (IS) fraction, and levels of input were adjusted using a pan-ACTIN Ab that reacts equally with both forms.

Unexpectedly, given that the vast majority of Actin-binding proteins associate with F-Actin (27, 28), ARID3A partitioned almost exclusively within the unpolymerized, G-Actin soluble fraction (Fig. 3A). These unexpected results encouraged us to analyze Actin-associated partitioning of a highly conserved, ARID3A paralogue, ARID3C (29). ARID3C is significantly condensed relative to ARID3A (S-Fig. 6), but both share all functional domains and undergo nuclear-cytoplasmic shuttling, with a fraction of ARID3C also localizing within lipid rafts following BCR stimulation (26). These shared features provided the opportunity to readily and more broadly investigate essential sequences required for G-Actin partitioning

SUMO-modification does not control ARID3 partitioning within G-ACTIN. We previously demonstrated that for $B C R$ signaling, ARID3A requires SUMOylation at a single, conserved lysine residue (K402) for exiting lipid rafts (18; S-Figure 5C). ARID3C requires SUMOylation of the equivalent K284 for rafts exit $(18,29)$. As shown in Figure 3B, both ARID3A-K402A and ARID3C-K284A fractionate primarily within the soluble fraction. Thus, SUMOylation plays no major role in G-Actin association.

The REKLES- $\beta$ domain is essential for ARID3A association within G-Actin. The REKLES domain is named for a hexapeptide (boxed in S-Fig. $5 \mathrm{~A}$ ) that is conserved among all ARID paralogues $(30,31)$. ARID3C encodes an additional a splice variant (termed ARID3C $\Delta 6)$ which lacks the C-terminal portion of the highly conserved protein-protein interaction (REKLES- $\beta$; exon 6; S-Fig. 6). ARID3C $\Delta 6$ fails to undergo nuclear-cytoplasmic shuttling nor does it associate with ARID3A in solution (29). Yet it does bind to common ARID3A DNA binding sites in vitro (29). As shown in Figure 3B, loss of this portion of the REKLES domain results in transfer of ARID3C $\triangle 6$ to the insoluble fraction.

Sequence alignments allow the REKLES domain to be divided into three sub-domains: A conserved 17 amino acid N-terminal REKLES- $\alpha$, a relatively conserved 51 amino acid Spacer-region and a highly conserved 59 amino acid C-terminal REKLES- $\beta$ domain (S-Fig. 5A, B). REKLES- $\alpha$ is required for nuclear localization (NLS) and REKLES- $\beta$ is essential for nuclear export (NES) $(18,30)$. REKLES- $\beta$ also is essential for ARID3A and ARID3C heteromeric interactions $(29,31)$.

The ARID3C $\triangle 6$ observation suggested that the corresponding region of ARID3A, the REKLES- $\beta$ domain, is critical for ARID3A residence within G-Actin. To test, we first determined if ARID3A carrying a complete deletion (d) (amino acids 500-562; S-Fig. 5C) of the 63 residue REKLES- $\beta$ was also transferred to the soluble G-Actin fraction. Accordingly, only $\sim 40 \%$ of REKLES $\beta$-deficient d500-562 remained within the G-Actin fraction. REKLES- $\beta$ amino acids 521-541 contain a cluster of residues (G532, Y535, G537, 
and L539) conserved in all ARID3 paralogues (S-Fig. 5B; 27). Alanine substitutions within each abolished G-protein soluble fraction association to varying degrees.

The REKLES- $\beta$ domain is essential for EZRIN-ARID3A interaction. The data above suggested that ARID3A interaction within PM G-Actin is mediated through its REKLES- $\beta$ subdomain, potentially via interaction with the cytoskeletal linker protein EZRIN. This hypothesis was tested by the Co-Immunoprecipitation (Co-IP) experiments of Figure 4. We transfected COS7 cells with full-length ARID3A (residues 1-601) or with each of the following REKLES mutations: d453-500, in which the entire REKLES domain is deleted (d); d500-521, in which the C-terminal 21 residues of the REKLES- $\alpha$ -

REKLES- $\beta$ Spacer reside just N-terminal to the REKLES- $\beta$ domain is deleted; 1 -541, in which ARID3A is truncated ( $t$ ) just N-terminal to REKLES- $\beta$; and d521-541, in which the REKLES- $\beta$ domain is deleted (Fig. 4A).

Transfected lysates were immunoprecipitated with a polyclonal anti-ARID3A Ab which previously had been shown to pull-down each of the input mutants $(30,31)$. After adjusting for equivalent levels of immunoprecipitated ARID3A inputs (Fig. 4B, middle panel), WT and mutant lysates were fractionated on SDS-PAGE and then Western blotted with an EZRIN monoclonal (m) Ab. As shown in Figure 4B, EZRINARID3A interaction was abrogated only by deletion of the REKLES- $\beta$ domain and $N$ terminal proximal section of the Spacer region.

Collectively these results indicate to us that ARID3A is tethered to G-Actin via interaction of its RECKLES- $\beta$ and proximal Spacer domain with EZRIN.

\section{SUMMARY}

To our knowledge, ARID3A was the first TF shown to function in lipid rafts (18), but its residence there is not unprecedented. Both STAT1 and STAT3 (33) as well as an isoform of the IgH coactivator OCA_B $(34,35)$ localize to lipid rafts. Several other TFs, including TFII_I, form cytoplasmic complexes (1417, 36). That TFs function directly or as intermediates in receptor signaling is supported by the association of p35-OcaB with galectin-1 (34) and, in this report, ARID3A with EZRIN.

Previous reports suggested that EZRIN binds specifically to F-Actin, which in turn, increases BCR signaling strength by aggregating and releasing lipid rafts from the cortical Actin cytoskeleton $(11,12)$. Here we have extended these results by the observation that an ARID3A-EZRIN-Actin interaction occurs exclusively within lipid rafts, and they are co-discharged from rafts following BCR stimulation (Figs. 1, 2, S-Fig. 2). Our data further indicate that, within lipid rafts, EZRIN links ARID3A indirectly to the cytoskeleton to control BCR signaling strength (Figs. 1, 2). But unexpectedly, ARID3A associates almost 
exclusively with unpolymerized G-Actin (Fig 3A, B). The interaction requires a patch of 5 residues conserved among ARID3 paralogues (S-Fig. 5B) within the 59 residue carboxyl terminal ( $\beta$ subregion) of the multifunctional REKLES domain. In addition, REKLES- $\beta$, as well as the C-terminal 21 residues of the REKLES- $\alpha \beta$ Spacer, link ARID3A to EZRIN (Fig. 4B).

These results add another layer of complexity to the ARID3A REKLES domain. The REKLES- $\alpha$ subregion encodes an NLS and a binding site for SUMO-I, whereas its $\beta$ subregion provides essential residues for nuclear export as well as for heteromeric interactions with EZRIN, BTK, PIAS-1 and UBC9 $(18,29-31)$. The 3-dimensional structure of the DNA binding domain of ARID3A has been solved $(37,38)$. Yet the structure of REKLES remains to be determined. The observed extent of REKLES multifunctionality as well as the architecture of the platform tethering G-Actin to the REKLES-EZRIN complex is a future goal of our research.

We have combined several of these features into the model of Figure $4 \mathrm{C}$. We suggest that a functional consequence of ARID3A discharge from lipid rafts is to release lipid rafts-associated G-Actin for polymerization to F-Actin. The extent to which this release is achieved contributes to the signaling threshold of the BCR. Conversely, reassembly of the polymerized F-Actin cytoskeleton might act to stabilize interactions among the BCR and additional signaling molecules via trapping the lipid raftlocalized signaling complex. In support, TIRFM studies (capable of observing both the BCR, Actin and EZRIN simultaneously) revealed EZRIN-Actin networks in resting B cells (39). That Ag-Ab binding induces transient EZRIN dephosphorylation followed by detachment of lipid rafts from the Actin cytoskeleton (39) is consistent with our observations (Figs.1,2) in respect to promotion of BCR-lipid raft interactions. We found it interesting in this regard that ARID3A and EZRIN share a binding partner, S100P. Dimeric S100P binds to and activates EZRIN by unmasking its F-Actin binding sites (40), whereas monomeric S100P represses the DNA-binding and transactivation activity of ARID3A (32).

We feel that further experimental refinement of our model is justified from a health perspective. For example, Diffuse Large B Cell Lymphoma (DLBCL)—both of the Activated $\underline{B}$ Cell $(A B C)$ and Germinal Center (GC) subtypes_co-segregate with high levels of phospho-EZRIN (41). These results implicate the cytoskeletal network in general, and EZRIN, in particular, in leukemia pathogenesis. Previously we demonstrated that ARID3A is highly expressed in ABC-DLBCL (42). Further, shRNA-mediated ARID3A $K D$ in ABC-DLBCL tumors, but not in GC-DLBCL subsets, led to loss of tumor growth and proliferation (42). While far less is known about ARID3C, RNA-Seq analyses recently identified it within a signaling complex consisting of Protein Tyrosine Phosphatase Receptor type R (PTPRR), $\alpha$-catenin, $\beta$-catenin and E-cadherin that formed exclusively in ovarian cancer (43). These data suggest that modulation of EZRIN, ARID3A, and potentially, ARID3C may provide both prognostic and therapeutic options for these and other malignancies. 


\section{ACKNOWLEGEMENTS}

We thank June Harriss, Debora Lerner, Chhaya Das and Maya Ghosh for help in cell culture and molecular techniques. We thank members of the Tucker laboratory for discussions and reading of the manuscript. We received outstanding experimental support at the MD Anderson Smithville Core Facilities, directed by Dr. Jianjun (J-J) Shen, from the following employees: Ms Luis Coletta, Ms Melissa Simper, Dr. Yueping Chen, Ms. Yoko Takata and Dr. Carol Mikulec. H.O.T. received support from NIH Grant R01CA31534, Cancer Prevention Research Institute of Texas (CPRIT) Grants RP100612, RP120348; and the Marie Betzner Morrow Centennial Endowment.

\section{ARTHUR CONTRIBUTIONS}

CS and HOT designed research; CS, LC, JT and DK performed research; CS and HOT analyzed data; HOT wrote the manuscript.

\section{COMPETING FINANCIAL INTERESTS}

The authors declare no competing financial interests

\section{REFERENCES}

1. Zheng B, Han M, Bernier M, Wen JK. Nuclear Actin and Actin-binding proteins in the regulation of transcription and gene expression. FEBS J. 2009; 276(10):2669-2685. Doi:10.1111/j.17424658.2009.06986.x

2. Lodish H, Berk A, Zipursky SL, Matsudaira P, Baltimore D, Darnell J. The Actin cytoskeleton. In: Molecular Cell Biology 4th edition. New York: W. H. Freeman; 2000.

3. G. Salbreux, G. Charras, E. Paluch. Actin cortex mechanics and cellular morphogenesis. Trends Cell Biol. 2012 Oct; 22(10):536-45. Doi: 10.1016/j.tcb.2012.

4. P. Chugh, E.K. Paluch. The Actin cortex at a glance. J. Cell Sci., 131 (14) (2018 Jul 15), jcs1862545 
5. S.J. Winder, K.R. Ayscough. Actin-binding proteins. J Cell Sci. 2005 Feb 15; 118(Pt 4):651-4.Doi: 10.1242/jcs.01670..

6. S.A. Freeman, A. Vega, M. Riedl, R.F. Collins, P.P. Ostrowski, E.C. Woods, et al. Transmembrane Pickets connect cyto- and pericellular skeletons forming barriers to receptor engagement. Cell. 201801 11; 172(1-2):305-317.e10 Doi.org/10.1016/j.cell.2017.12.023.

7. Li J. Yin W., Jing Y., Kang D., Yang L. et al. The coordination between B Cell Receptor signaling and the Actin cytoskeleton during B cell activation. Frontiers in Immunol. 2019. 9: 1664-3224.

$\mathrm{DOI}=10.3389 /$ fimmu.2018.03096.

8. Pore D, Bodo J, Danda A, Yan D, Phillips JG, Lindner D, et al. Identification of Ezrin-RadixinMoesin proteins as novel regulators of pathogenic B-cell receptor signaling and tumor growth in diffuse large B cell lymphoma. Leukemia (2015) 29:1857-67. Doi: 10.1038/leu.2015.86.

9. Ponuwei GA, A glimpse of the ERM proteins. J Biomed Sci. (2016) 23:35. Doi: 10.1186/s129290160246-3.

10. Gupta N, Wollscheid B, Watts JD, Scheer B, Aebersold R, DeFranco AL. Quantitative proteomic analysis of $B$ cell lipid rafts reveals that Ezrin regulates antigen receptor-mediated lipid raft dynamics.

Nature Immunol. (2006) 7:625-33. Doi: 10.1038/ni1337.

11. Treanor B, Depoil D, Bruckbauer A, Batista FD. Dynamic cortical Actin remodeling by ERM proteins controls BCR microcluster organization and integrity. J Exp Me. (2011) 208:1055-68. Doi: 10.1084/jem.20101125.

12. Hao S., August A. 2005. Actin depolymerization transduces the strength of B-cell receptor stimulation. Mol. Biol. Cell. 16:2275-2284 10.1091/mbc.E04-10-0881.

13. Zheng B, Han M, Bernier M, Wen JK. Nuclear Actin and Actin-binding proteins in the regulation of transcription and gene expression. FEBS J. 2009; 276(10):2669-2685. Doi:10.1111/j.17424658.2009.06986.x.

14. Miralles F, Posern G, Zaromytidou Al, Treisman R. Actin dynamics control SRF activity by regulation of its coactivator MAL. Cell. 2003 May 2; 113(3):329-42. Doi: 10.1016/s0092-8674(03)002782.

15. Posern G, Miralles F, Guettler S and Treisman R. Mutant actins that stabilize F-Actin use distinct mechanisms to activate the SRF coactivator MAL. EMBO J. 2004; 23:3973-3983. Doi: 10.1038/sj.emboj.7600404

16. Haller K, Rambaldi I, Daniels E, Featherstone M. Subcellular localization of multiple PREP2 isoforms is regulated by Actin, tubulin, and nuclear export. J Biol Chem. 2004; 279:49384-49394. Doi: 10.1074/jbc.M406046200 
17. Favot L, Hall SM, Haworth SG, Kemp PR. Cytoplasmic YY1 is associated with increased smooth muscle-specific gene expression: implications for neonatal pulmonary hypertension. Lin X, Sime PJ, Xu $\mathrm{H}$, et al. Yin yang 1 is a novel regulator of pulmonary fibrosis. Am J Respir Crit Care Med. 2011; 183(12):1689-1697. Doi:10.1164/rccm.201002-02320C.

18. Schmidt C, Kim D, Ippolito GC, et al. Signaling of the BCR is regulated by a lipid rafts-localised transcription factor, Bright. EMBO J. 2009; 28(6):711-724. Doi:10.1038/emboj.2009.20.

19. Herrscher RF, Kaplan MH, Lelsz DL, Das C, Scheuermann R, Tucker PW. The immunoglobulin heavy-chain matrix-associating regions are bound by Bright: a B cell-specific trans-activator that describes a new DNA-binding protein family. Genes Dev. (1995) 24:3067-82. Doi:

10.1101/gad.9.24.3067.

20. Ratliff ML, Templeton TD, Ward JM, Webb CF. The bright side of hematopoiesis: regulatory roles of ARID3a/bright in human and mouse hematopoiesis. Front Immunol. (2014) 5:113. Doi: 10.3389/fimmu.2014.00113.

21. Saeki K, Miura Y, Aki D, Kurosaki T, Yoshimura A. The B cell-specific major raft protein, Raftlin, is necessary for the integrity of lipid raft and BCR signal transduction. EMBO J. 2003; 22(12):3015-3026. Doi:10.1093/emboj/cdg293

22. Sproul TW, Malapati S, Kim J, Pierce SK. Cutting edge: B cell antigen receptor signaling occurs outside lipid rafts in immature B cells. 2000, 165 (11) 6020-6023; Doi: https://doi.org/10.4049/jimmunol.165.11.6020

23. Putnam MA, Moquin AE, Merrihew M, Outcalt C, Sorge E, Caballero A, Gondré.Lewis TA, Drake JR. Lipid raft.independent $B$ cell receptor_mediated antigen internalization and intracellular trafficking. 2003 Jan; 170(2):905-912. DOI: 10.4049/jimmunol.170.2.905.

24. Pollard, TD. Actin and Actin-Binding Proteins. Cold Spring Harbor perspectives in biology vol. 8, 8 a018226. 1 Aug. 2016, doi:10.1101/cshperspect.a018226.

25. Clarke M, Spudich J. Nonmuscle contractile proteins: The role of Actin and myosin in cell motility and shape determination. Ann. Rev. Biochem. 1997; 46:797-822. 10.1146/annurev.bi.46.070177.004053.

26. No SY, Gunning P, Eddy R, Ponte P, Leavitt J, Shows T, Kedes L. Evolution of the functional human beta-Actin gene and its multi-pseudogene family: Conservation of noncoding regions and chromosomal dispersion of pseudogenes. Mol. Cell. Biol. 1985. Oct; 5(10):2720-2732. DOI: 10.1128/mcb.5.10.2720

27. 27. Gressin, L., Guillotin, A., Guérin, C., Blanchoin, L. and Michelot, A. Architecture dependence of Actin filament network disassembly. Curr. Biol., https://doi.org/10.1016/j.cub.2015.04.011 (2015). 
28. Keren, K., Yam, P. T., Kinkhabwala, A., Mogilner, A. and Theriot, J. A. Intracellular fluid flow in rapidly moving cells. Nat. Cell Biol., https://doi.org/10.1038/ncb1965 (2009).

29. Tidwell JA, Schmidt C, Heaton P, Wilson V, Tucker PW. Characterization of a new ARID family transcription factor (Brightlike/ARID3C) that co-activates Bright/ARID3A-mediated immunoglobulin gene transcription. Mol Immunol. 2011; 49(1-2):260-272. doi:10.1016/j.molimm.2011.08.025

30. Kim D, Tucker PW. A regulated nucleocytoplasmic shuttle contributes to Bright's function as a transcriptional activator of immunoglobulin genes. Mol Cell Biol. 2006 Mar; 26(6):2187-201.

31. Kim D, Probst L, Das C, Tucker PW.REKLES is an ARID3-restricted multifunctional domain. J Biol Chem. 2007 May 25; 282(21):15768-77.

32. Zong RT, Das C, Tucker PW. Regulation of matrix attachment region-dependent, lymphocyte restricted transcription through differential localization within promyelocytic leukemia nuclear bodies.

EMBO J. 2000 Aug 1; 19(15):4123-33. Doi: 10.1093/emboj/19.15.4123.

33. Sehgal, P.B., Guo, G.G., Shah, M., Kumar, V. \& Patel, K. Cytokine signaling: STATS in plasma membrane rafts. J Biol Chem 2002 Apr; 277(14):12067-12074. DOI: 10.1074/jbc.m200018200.

34. Yu, X., Siegel, R. \& Roeder, R.G. Interaction of the B cell-specific transcriptional coactivator OCA$B$ and galectin-1 and a possible role in regulating BCR-mediated B cell proliferation. J Biol Chem 281, 15505-15516 (2006). Doi: 10.1074/jbc.M509041200

35. Yu, X., Wang, L., Luo, Y. \& Roeder, R.G. Identification and characterization of a novel OCA-B isoform. Implications for a role in B cell signaling pathways. Immunity 14, 157-167 (2001). https://doi.org/10.1016/S1074-7613(01)00099-1

36. Caraveo G, van Rossum DB, Patterson RL, Snyder SH, Desiderio S (2006). Action of TFII-I outside the nucleus as an inhibitor of agonist-induced calcium entry. Science. 314(5796), 122-125. https://Doi.org/10.1126/science.1127815.

37. Liu G, Huang YJ, Xiao R, Wang D, Acton TB, Montelione GT. Solution NMR structure of the ARID domain of human AT-rich interactive domain-containing protein 3A: A human cancer protein interaction network target. Proteins. 2010; 78(9):2170-2175. Doi:10.1002/prot.22718.

38. Invernizzi G, Tiberti M, Lambrughi M, Lindorff-Larsen K, Papaleo E. Communication routes in ARID domains between distal residues in helix 5 and the DNA-binding loops. PLoS Comput Biol. 2014 Sep; 10(9): e1003744. Doi: 10.1371/journal.pcbi.1003744.

39. Gupta N, Wollscheid B, Watts JD, Scheer B, Aebersold R, DeFranco AL. Quantitative proteomic analysis of $B$ cell lipid rafts reveals that Ezrin regulates antigen receptor-mediated lipid raft dynamics. Nature Immunol. (2006) 7:625-33. Doi: 10.1038/ni1337.

40. Koltzscher M, Neumann C, König S, Gerke V. Ca2+-dependent binding and activation of dormant ezrin by dimeric S100P. Mol Biol Cell. 2003 Jun; 14(6):2372-84. Doi: 10.1091/mbc.e02-09-0553. 
41. 41. Pore D, Bodo J, Danda A, Yan D, Phillips JG, and Lindner D, et al. Identification of EzrinRadixin Moesin proteins as novel regulators of pathogenic B-cell receptor signaling and tumor growth in diffuse large B-cell lymphoma. Leukemia (2015) 29:1857-67. Doi: 10.1038/leu.2015.86.

42. Deng W, Dekker JD and Tucker HO. FOXP1 and ARID3A collaborate to activate transcription of core embryonic stem cell factors in Activated B-Cell Diffuse Large B cell Lymphoma. J Embryol Stem Cell Res 2018, 2(1): 000110. ISSN: 2640-2637.

43. Wang Y, Cao J, Liu W, Zhang J, Wang Z, Zhang Y, Hou L, Chen S, Hao P, Zhang L, Zhuang M, Yu Y, Li D, Fan G. Protein tyrosine phosphatase receptor type R (PTPRR) antagonizes the Wnt signaling pathway in ovarian cancer by dephosphorylating and inactivating $\beta$-catenin. J Biol Chem. 2019 Nov 29; 294(48):18306-18323. Doi: 10.1074/jbc.RA119.010348.

43. McConnell FM, Shears SB, Lane PJ, Scheibel MS, Clark EA. Relationships between the degree of cross-linking of surface immunoglobulin and the associated inositol 1,4,5-trisphosphate and $\mathrm{Ca2+}$ signals in human B cells. Biochem J. 1992; 284 (Pt 2)(Pt 2):447-455. Doi: 10.1042/bj2840447.

44. Wang F, Gregory C, Sample C, et al. Epstein-Barr virus latent membrane protein (LMP1) and nuclear proteins 2 and $3 \mathrm{C}$ are effectors of phenotypic changes in B lymphocytes: EBNA-2 and LMP1 cooperatively induce CD23. J Virol. 1990;64 (5):2309-2318. doi:10.1128/JVI.64.5.2309-2318.1990

45. Cerimele F, Battle T, Lynch R, Frank DA, Murad E, Cohen C, Macaron N, Sixbey J, Smith K, Watnick RS, Eliopoulos A, Shehata B, Arbiser JL (2005) Reactive oxygen signaling and MAPK activation distinguish Epstein Barr Virus (EBV)-positive versus EBV-negative Burkitt's lymphoma. Proc Natl Acad Sci USA. January 4, 2005102 (1) 175-179; https://Doi.org/10.1073/pnas.0408381102.

46. Laskov R, Berger N, Scharff MD, Horwitz MS. Tumor necrosis factor-alpha and CD40L modulate cell surface morphology and induce aggregation in Ramos Burkitt's lymphoma cells. Leukemia \& Lymphoma.

2006 Mar; 47(3):507-519. DOI: 10.1080/10428190500221454.

47. Kuhn JR, Poenie M. Dynamic polarization of the microtubule cytoskeleton during CTL-mediated killing. Immunity.16 (1). 2002. 111-12116: https://doi.org/10.1016/S1074-7613(02)00262-5

48. Riddell A, Gardner R, Perez-Gonzalez A, Lopes T, Martinez L. Rmax: A systematic approach to evaluate instrument sort performance using center stream catch. Methods. 2015; 82:64-73. doi:10.1016/j.ymeth.2015.02.017.

\section{MATERIALS AND METHODS}

Cell lines. Raji (EBV+, 45); Daudi (EBV+, 45); Ramos (EBV-, 46) and CL01 (EBV-, 47)_were obtained from ATCC (Manassas, Virginia) and maintained as previously described (18). Cells were grown and maintained in either Dulbecco's modified Eagle medium (DMEM) supplemented with 10\% fetal bovine 
serum (FBS; Invitrogen) or in RPMI medium containing 10\% FBS. CD43- B cells were prepared by negative selection of whole human blood (Gulf Coast Regional Blood Center, Houston, Texas) or from 10-wk-old BALB/c murine splenocytes (19 30).

Constructs. Mutant forms of ARID3A and ARID3C used in this study were generated previously by site directed mutagenesis $(18,29-31)$.

Preparation of stable sh-RNA retrovirally transduced B cell lines. Stable transductants were established by employment of the Phoenix-A retroviral system. Approximately $3 \times 10^{5}$ amphitrophic Phoenix-A packaging cells in $4 \mathrm{ml}$ of DMEM were supplemented with $10 \%$ fetal bovine serum (FBS) in 60-mm plates. After one day of culture, cells were transfected using FuGene6, and viral supernatant was harvested 2 days post-transfection, centrifuged, and filtered to remove live cells and debris. The target cell lines described above were plated $\left(3 \times 10^{5}\right)$ onto $60-\mathrm{mm}$ plates and growth medium was replaced with viral mixture. To introduce EZIN-knockdown sequences, we used oligos ordered from Integrated DNA Technologies with restriction site overhangs Bbsl and Xhol (sense, 5'-ACCG GCCGTGGAGAGAGAGAAAGATTCAAGAGATCTTTCTCTCTCTCCACGGCTTTTTTACCGGTC-3';

and anti-sense, 5'-TCGAGACCGGTAAAAAAGCCGTGGAGAGAGAGAAAGATCTCTTGAATCTTT CTCTCTCTCCACGGC-3'). Stable lines were established by selection with $2 \mu \mathrm{g} / \mathrm{ml}$ of puromycin from day 2 post-infection.

B-cell stimulation. To measure signaling effects at low doses of anti-lgM where receptor internalization is minimized, we used monoclonal anti-lgM antibodies in the absence of secondary crosslinking. To stimulate B cells, $500 \mathrm{ng}$ of $\left.\mathrm{F}(\mathrm{ab})_{2}\right)_{2}$ fragments of $\alpha-\mu$ (clone JDC-15; Dako [a-human];

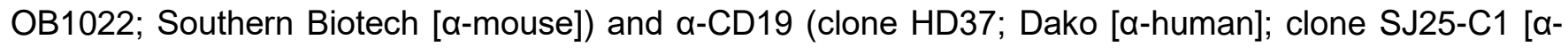
mouse]) were added to $5 \times 10^{8}$ cells for $5 \mathrm{~min}$ at $37^{\circ}$. We determined by FACS analysis and semiquantitative western of lipid raft-associated mlgM (data not shown and S-Figure 1) that under these conditions $\sim 1-5 \%$ of $\mathrm{mlgM}$ in rafts and membranes are engaged.

Preparation of lipid rafts. Approximately $500 \mathrm{mg}$ of wet cell pellet were washed twice in ice-cold phosphate buffered solution (PBS) and homogenized in $5 \mathrm{ml}$ of $10 \mathrm{mM}$ Tris/Cl (pH 7.4), $1 \mathrm{mM}$ EDTA, 250 $\mathrm{mM}$ sucrose, $1 \mathrm{mM}$ phenylmethylsulfonyl fluoride and $1 \mu \mathrm{g} / \mathrm{ml}$ leupeptin (all from Sigma, St Louis, MO) in a tightly fitted Dounce homogenizer using five strokes. The resulting homogenate was centrifuged at 900 $g$ for $10 \mathrm{~min}$ at $4^{\circ} \mathrm{C}$, the resulting supernatant was then subjected to centrifugation at $110000 \mathrm{~g}$ for $90 \mathrm{~min}$ at $\left.4^{\circ} \mathrm{C}\right)$. The resulting membrane pellet was resuspended in ice cold $500 \mu \mathrm{l}$ TNE buffer (10 mM Tris/Cl [pH 7.4], 150 mM NaCl, 5 mM EDTA, 1\% Triton X-100 [Sigma], 10X protease inhibitors [Complete tablets, Roche, Indianapolis, IN]). Sucrose gradients for the preparation of lipid rafts were assembled previously described (18). Lipid rafts were isolated by flotation on discontinuous sucrose gradients. 
Membrane pellets were extracted for $30 \mathrm{~min}$ on ice in TNE buffer. For the discontinuous sucrose gradient, $1 \mathrm{ml}$ of cleared supernatant was mixed with $1 \mathrm{ml}$ of $85 \%$ sucrose in TNE and transferred to the bottom of an ultracentrifugation tube, followed by overlay with $6 \mathrm{ml}$ of $35 \%$ sucrose in TNE and $3.5 \mathrm{ml}$ of $5 \%$ sucrose in TNE. Samples were spun at $200,000 \mathrm{~g}$ for $30 \mathrm{~h}$ at $4^{\circ} \mathrm{C}$; fractions were collected from the top of the gradient and analyzed using Western blotting and/or coimmunoprecipitation), as described (30, 31).

Immunoprecipitation/Western analyses. We employed a stringent RIPA formulation of $500 \mathrm{mM}$ $\mathrm{NaCl} ; 10 \mathrm{mM}$ Tris-Cl pH 8; 0.1\% SDS; 5 mM EDTA, pH 8; 10X protease inhibitor (Complete tablet, Roche) to solubilize lipid rafts for subsequent immunoprecipitation experiments. Briefly, buoyant fractions, taken from the discontinuous gradient centrifugation, were pooled and incubated with the same volume of RIPA buffer on ice for $15 \mathrm{~min}$. Resulting extracts were pre-cleared by rocking with $1 \mathrm{ml}$ of a $5 \%$ slurry of RIPA equilibrated Protein A beads CL-4B (Amersham Pharmacia, Uppsala) for $4 \mathrm{~h}$ at $4^{\circ} \mathrm{C}$ and removal of the precipitate. The resulting supernatant was then subjected to IP/western assays. The following antibodies were used: $\alpha$-CD19 (clone 6D5, Dako), $\alpha$-ARID3A polyclonal Ab (produced in house; 19), $\alpha$ IgM (BD Pharmingen), a-V5 (Sigma), a-Raftlin (graciously provided by Dr. Akihiko Yoshimura; 21), $\alpha-$ SUMO-1 (Sigma), anti-TFII-I (kindly provided by Dr. Carol Webb,18); pan a-Actin (rabbit origin, Cytoskeleton, Inc), a-Acid sphingomyelinase (SMPD1; ab83354, Abcam), goat a-ezrin (sc-6407; Santa Cruz)

Accumulation of cytosolic calcium. Indo-1 AM (acetoxymethyl ester; Invitrogen) was added to $3 \times 10^{6}$ leukemia/lymphoma cells in $500 \mu$ l Hank's Balanced Salt Solution (HBSS; Invitrogen) and 10\% FCS (HBSS-10). The Indo-1 final concentration was $1 \mu \mathrm{M}$. Following incubation at $37^{\circ} \mathrm{C}$ for $30 \mathrm{~min}$, cells were kept at RT for 5 min and then washed with HBSS containing 2 mM HEPES buffer and no serum $(\mathrm{HBSH}-0)$. All manipulations including the incubation were in the dark. Cells were then reacted with a rat anti-human CD16/CD32 Fc blocking Ab (clone 2.4G2, Pharmingen) and washed with HBSH-0 in the dark at $4^{\circ} \mathrm{C}$. The cells were resuspended in $50 \mu \mathrm{HBSH}-0$ added to a total volume of $\sim 150 \mu \mathrm{l}$. Prior to Ca2+ analyses, cells were filtered at RT, warmed to $37^{\circ} \mathrm{C}$ and then placed in a flow cytometer maintained at $37^{\circ} \mathrm{C}$ at a flow rate of 250 cells/s. Anti-IgM and anti-CD19 were added (detailed in above in "B Cell stimulation") $30 \mathrm{~s}$ prior to initiation of the experiments. Data acquisition employed a 30 -s baseline and was continued for $300 \mathrm{~s}$ at $37^{\circ} \mathrm{C}$. Cells were analyzed at $\sim 250$ cells/s, and their flux in calcium concentration was determined by $485 / 405 \mathrm{~nm}$ emission ration with excitation at $355 \mathrm{~nm}$. Calibration was performed by measuring Rmin and Rmax, and applying the equation described previously (48). Responses are reported as [Ca2] vs time.

Purification of Actin. To obtain detergent-soluble (Actin-free) and insoluble (Actin-enriched) fractions, ARID3A-transfected COS7 cells were harvested by scraping, and then resuspended in $750 \mu \mathrm{l}$ 
of pre-warmed LAS buffer [50 mM Pipes ( $\mathrm{pH}$ 6.9), $50 \mathrm{mM} \mathrm{NaCl}, 5 \mathrm{mM} \mathrm{MgCl}$, $5 \mathrm{mM} \mathrm{EGTA,} \mathrm{5 \%} \mathrm{(v/v)}$ glycerol, $0.1 \%$ NP-40, $0.1 \%$ Triton X-100 (with or without $0.1 \%$ Tween 20 ), $0.1 \% \beta$-mercaptoethanol, 1 mM ATP, $0.001 \%$ Antifoam C, and a protease inhibitor cocktail consisting of $0.4 \mathrm{mM}$ tosyl arginine methyl ester, $1.5 \mathrm{mM}$ leupeptin, $1 \mathrm{mM}$ pepstatin $\mathrm{A}$, and $1 \mathrm{mM}$ benzamidine]. Cells were lysed by 10 passages through a 25-gauge needle. The lysate was clarified by centrifugation ( $400 \mathrm{~g}$ for $5 \mathrm{~min}$ at RT). The supernatant $(100 \mu \mathrm{l})$ was collected, and Actin polymerization was initiated by addition of $2 \mathrm{mM} \mathrm{MgCl}, 0.2$ $\mathrm{mM}$ EGTA, $100 \mathrm{mM} \mathrm{KCl}$, and $1 \mu \mathrm{M}$ phalloidin and by incubation for 1 hour at $37^{\circ} \mathrm{C}$. F-Actin and F-Actinbinding proteins were pelleted by ultracentrifugation $(270,000 \mathrm{~g})$ for 1 hour at $4^{\circ} \mathrm{C}$. The supernatant (GActin fraction) was collected, and the pellet (F-Actin fraction) was washed twice with Milli-Q water and resuspended in $100 \mu \mathrm{l}$ of $8 \mathrm{M}$ urea. After addition of SDS loading buffer, the samples were separated by SDS-polyacrylamide gel electrophoresis (PAGE) and analyzed by Western blotting for Actin using antipan-Actin polyclonal rabbit Ab (1:2000; Cell Signaling Technology, 4968).

Immunofluorescence staining of murine B cells. Mouse CB7BI/6 MZ and FO B cells were isolated from mouse spleen cell suspension by anti-CD19 exclusion using a kit \#130-100-366 (Miltenyi Biotec). Cells were fluorescently stained with rabbit $\alpha \beta$-Actin mAb (SP124; ab115777; Abcam) developed with goat anti-rabbit lgG H\&L (Alexa Fluor® 594), mouse anti-ARID3A mAb A-4 (sc-398367, Santa Cruz Biotechnology) developed with goat anti-mouse IgG H\&L (Alexa Fluor® 488; ab150117, Abcam) and stained with DAPI (Staining Solution ab228549, Abcam). Visualization is described below and shown in Figure 2B and S-Figure 4.

Multiphoton microscopy (MPM) imaging. MPM imaging as detailed by Kuhn and Poenie (48) was performed at a rate of one to two processed frames per second. Each image in the resulting MPM movie sequence was enhanced slightly using a $3 \times 3$ convolution kernel of [[-1/2 1/2 1/2], [-1 1/2 1/2], $\left[\begin{array}{lll}-1 / 2 & 1 / 2 & 1 / 2\end{array}\right]$, which added a small emboss effect to the image and improved visibility. Fluorescent images were acquired using a 12 bit CCD camera (Model DVC-1312M, DVC, Austin, TX) on a Nikon Diaphot 200 fluorescence microscope. Image stacks were obtained using a MAC 2000 z-axis focus controller (Ludl Electronic Products, Hawthorne, NY) and a custom image acquisition plugin written for ImageJ. The point-spread function (PSF) was measured under similar conditions using $100 \mathrm{~nm}$ fluorescent beads (L-5473, Molecular Probes) diluted in $\mathrm{dH} 2 \mathrm{O}$ and dried on glass slides to give approximately one bead per field. Image stacks were deconvolved for 500 iterations using the expectation maximization algorithm in XCOSM (49). 3D projections were calculated using the maximum value projection method in ImageJ, and stereo pairs were generated using images that differed in rotation by 10 degrees. Microtubule images were enhanced using a multiscale 3D line filter implemented as a custom plugin for ImageJ. 


\section{FIGURE LEGENDS}

Figure 1. The ARID3A-EZRIN-complex is directed to different fates depending on the signaling threshold of the BCR. Raji, Ramos, CL01 and Daudi ( $10^{8}$ cells/dish) were stimulated for 5 min with (a)100 ng anti-IgM(a-lgM), (b) 100 ng a-CD19 or (c) 100 ng a-IgM +100 ng a-CD19. Lipid rafts (Raft), plasma membranes (Memb) and whole cell lysates (WCL) were analyzed following anti-ARID3A IP and Western blotting with anti-EZRIN. EZRIN-ARID3A do not Co-IP in either stimulated or nonstimulated WLC (lanes 5, 6, 11, 12, 17, 18, 23 and 24) but do within BCR-stimulated lipid rafts (lanes $\underline{8}$ and 20 in a and c). ARID3A-EZRIN Co-IP in lipid rafts of the higher threshold cell lines (Ramos and CL01) stimulated with anti-IgM only (a, lanes 8 and 20); within all cell lines stimulated with anti-CD19 only (b, lanes 2, 8, 14 and 20); and are discharged in all cell lines following anti-lgM + anti-CD19 stimulation (c, lanes 2, 8, 14 and 20). EZRIN is retained in the Memb only following strong co-stimulation (c, lanes

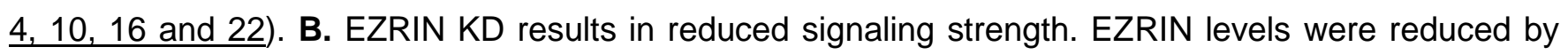
shRNA knockdown (KD; S-Fig 4) and calcium (Ca2+) flux was measured in the indicated B cell tumors of high or low signaling strength following strong costimulation as detailed in Materials and Methods. Data shown are representative of 3 independent experiments. $\alpha$-IgM + a-CD19 were added $30 \mathrm{~s}$ (upward arrow) before the beginning of the experiment, and the cells were analyzed at 250 cells/s. Results are plotted as the mean calcium concentration vs time. A significant difference was apparent between high vs low signaling cells when comparing their calcium response to stimulation with and without Ezrin knockdown.

Figure 2. Interaction and discharge of ARID3A-Actin complexes within lipid rafts as a function of BCR signal strength. A. ARID3A Co-IPs with a $\beta$-Actin in lipid rafts of B cell lines (lanes 1 , $\underline{5,9}$ and 13 ) regardless of their signaling strength. Strong stimulation ( $\alpha$-lgM + $\alpha$-CD19; lanes 4, 8, 12 , and 16) discharges ARID3A from ACTIN in lipid rafts of all cells, whereas weaker stimulation ( $\alpha$-CD19 or $\alpha-\lg M)$ discharged ACTIN from ARID3A in lipid rafts of strong (Raji and Daudi; lanes 2, 3, 14 and 15), but not weak (Ramos and CL-01; lanes 6, 7, 10 and 11) signaling strength B cells. Strong stimulation (lanes

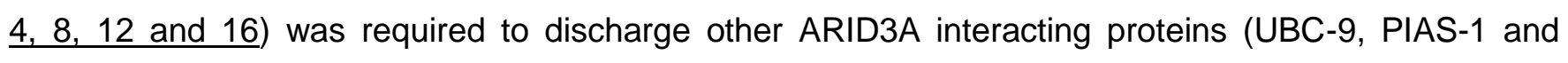
SUMOI) from lipid rafts. B. Triple immunofluorescence of murine B cells stained for Actin (green), ARID3A (red) and nuclei (blue) revealed strong Actin-ARID3A association (yellow; also illustrated in the movies of SFigure 4)

Figure 3. ARID3A and related paralogue, ARID3C localize within monomeric G-Actin via the conserved REKLES- $\beta$ domain. A. ARID3A and $3 C$ transfected COS7 lysates were processed via Triton 
X-100 and/or TritonX-100 + TWEEN-20 fractionation into insoluble (IS, F-Actin) or soluble (S, GActin) proteins. After fractionation by SDS-PAGE, samples were Western blotted with the indicated Abs; antiSMPD1 served as an IS positive control and Actin inputs were equalized with pan-Actin Ab. ARID3A and $3 C$ partitioned primarily within the G-Actin fraction. B. Loss of the REKLES domain (ARID3A-d(521541) and ARID3C $\Delta 6$ ), but not SUMO-I binding motifs (ARID3A-K402A or ARID3C-K284A) abolishes partitioning into the soluble G-Actin fraction. C. A region within REKLES- $\beta$ (amino acids 532-540 and indicated on S-Figure 5B) contains a cluster of conserved residues (G532, Y535, G537, and L539) whose point mutation significantly reduce or abolish ARID3A association with G-Actin.

Figure 4. REKLES- $\beta$ deletions abrogate ARID3A-EZRIN interaction. A. Diagram of ARID3A REKLES deletion (d) mutants. Deletions are indicated by gaps. B. EZRIN-ARID3A interaction was abrogated only by deletion ( $d 453-500$ and $d 521-541$ ) of the REKLES- $\beta$ domain. Full length ARID3A and its deletion mutants of $(A)$ were transfected into COS7 cells and lysates were immunoprecipitated with anti-ARID3A prior to resolution on SDS-PAGE followed by anti-EZRIN Western blotting (upper panel);

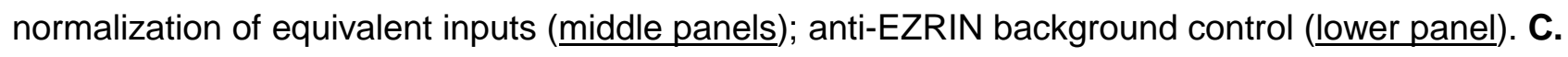

ARID3A is tethered to G-Actin via interaction of its REKLES- $\beta$ domain with EZRIN. Cartoon summarizing and extending the findings of this report. We speculate that when the ARID3A tetramer (large black spheres) interacts with EZRIN (green sphere) via a patch of conserved REKLES- $\beta$ residues (small black spheres with single amino acid codes in white) and just proximal Spacer residues (small red spheres) EZRIN is discharged from lipid rafts in order to release lipid rafts-associated ATP-G-Actin (brown semicircles), possibly for polymerization to ADP-F-Actin (brown chains).

\section{SUPPLEMENTAL FIGURE LEGENDS}

S-Figure 1. Biochemical fractionation indicates that lipid rafts, but not other subcellular compartments, vary among B cell tumors in levels of ARID3A. Exponentially growing Daudi and CL01 cells were fractionated into cytoplasm (CY), soluble nucleoplasm (NP), chromatin (CH) and nuclear matrix (NM) according to previous protocols $(18,27)$. Immunoblotting of equal aliquots $(\sim 25 \mu \mathrm{g})$ of protein with anti-ARID3A antiserum (Lanes 1-8) revealed no differences between these two cell lines. Cytoplasmic extracts (Lanes 1, 9, 5 and 12; 25 $\mu \mathrm{g}$ as equivalent to $\sim 2 \times 10^{5}$ cells) were used to normalize Western (Lanes 9-14,). Plasma membranes (PM; lanes 10 and 13) and lipid rafts (R; lanes 11 and 14) 
were prepared from $\sim 1 \times 10^{7}$ cells, as described above. The entire preparation was used for SDSPAGE/Western, which was internally normalized by reprobing the filter with anti-Raflin antiserum.

\section{S-Figure 2. Strength of BCR stimulation leads to differential lipid rafts discharge of ARID3A,} but not its interacting partner, SUMO-I. SUMO-I levels were assessed under conditions in which lipid rafts levels of ARID3A were modulated as described in the text and in Figure 1. A. Following moderate stimulation with anti-IgM, ARID3A is discharged from lipid rafts of Raji and Daudi cells (lanes $\underline{2}$ and 14), but not from those of Ramos and CL01 cells (lanes 8 and 20). B. ARID3A remains localized within rafts of all cell lines following weak stimulation by anti-CD19 (lanes 2, 8, 14 and 20). C. Under the strongest stimulatory condition (anti-lgM + anti-CD19), ARID3A is fully discharged (lanes 2, 8, 14 and 20). A-C. No change was observed in the expression levels of SUMO-1, which modifies ARID3A and ARID3C at single $\mathrm{K}$ residues (S-Figure $5 \mathrm{C}$ ) and interacts with ARID3A and ARID3C in lipid rafts (18)

.S-Figure 3. Ezrin retroviral knockdown levels. Levels of ezrin were determined following shEzrin and sh-Scrambled transduced lymphoma/leukemia cells via Western blotting as detailed in Methods and Materials. Data shown are representative of three independent experiments

S-Figure 4. Computerized 3D reconstruction demonstrate Actin-ARID3A interaction in B lymphocytes. Murine FO B cells were purified, stained with fluorescent-antibodies and imaged as detailed in Materials and Methods. Significant colocalisation (yellow) was observed for ARID3A (red) and Actin (green). DAPE-stained nuclei are shown in blue.

S-Figure 5. ARID3 family REKLES domain alignments. A. Schematic of ARID3A with domains denoted in colored boxes, including the Acidic, DNA binding (ARID) and multifunctional homomerization/nuclear export/EZRIN-interacting REKLES domain. B. The alignment of ARID3 REKLES- $\alpha$ subdomains of human (Homo sapiens), mouse (Mus musculus), chicken (Gallus gallus), pufferfish (Takifugu rubripes), and the urochordate tunicate Ciona (Ciona intestinalis) are shown. For the REKLES- $\beta$ alignment, additional sequences from mosquito (Anopheles gambiae), fruit fly (Drosophila melanogaster), nematode (Caenorhabditis elegans), and the crustacean Daphnia (Daphnia pulex) are indicated. Point mutants used in this study are denoted with a blue dot above the aligned sequence. C. A SUMO-I consensus motif with conjugated $K(\boldsymbol{\Delta})$ is located N-terminal to the REKLES domain at the indicated positions for ARID3A and ARID3C. Modified from references 29-31.

S-Figure 6. Schematic of ARID3A and ARID3C loci. Shown in scale (kb marker at bottom) are exons encoding ARID3A, ARID3C, and ARID3C $\triangle 6$. ARID $3 C$ and ARID3C $\triangle 6$ loci are compressed relatively to that of ARID3A. REKLES- $\alpha$ exons, red; REKLES- $\beta$ exons, gray. The dotted triangle indicates the alternative pre-mRNA skipping of exon 6. 
A

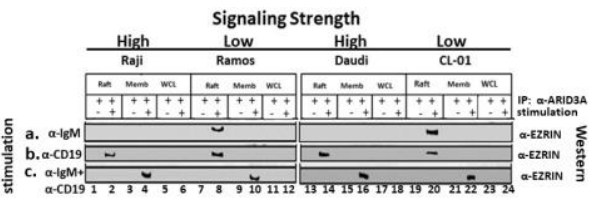

B

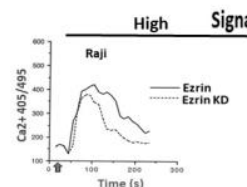

Time (s)
Daudi
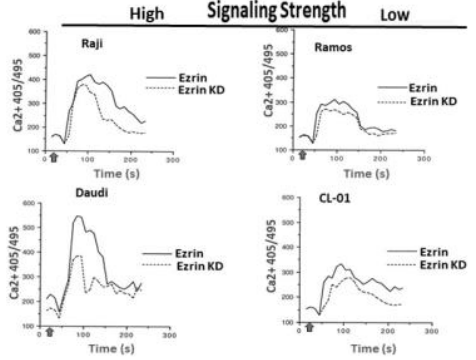

Figure 1

\section{Figure 2}

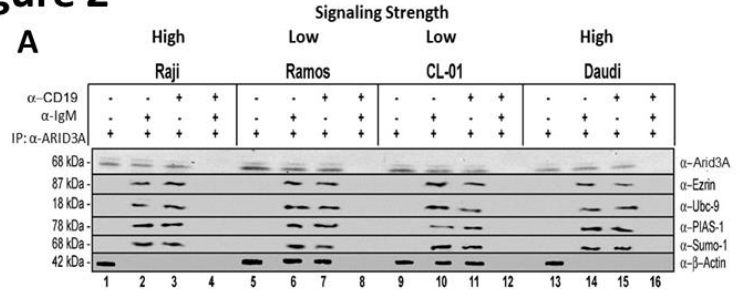

B

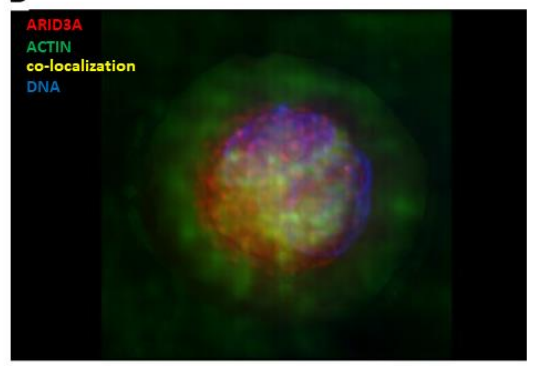

Figure 3

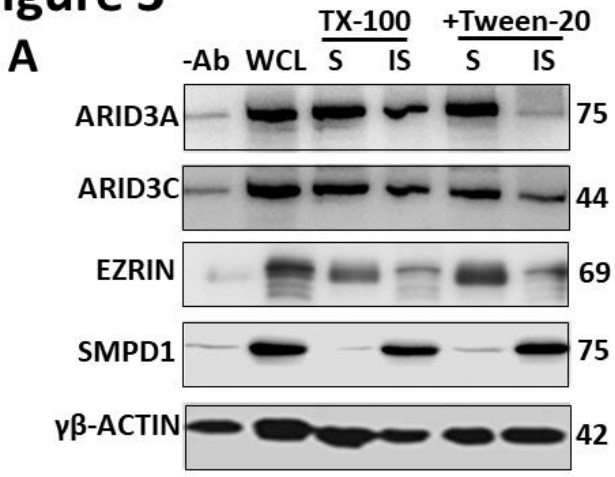

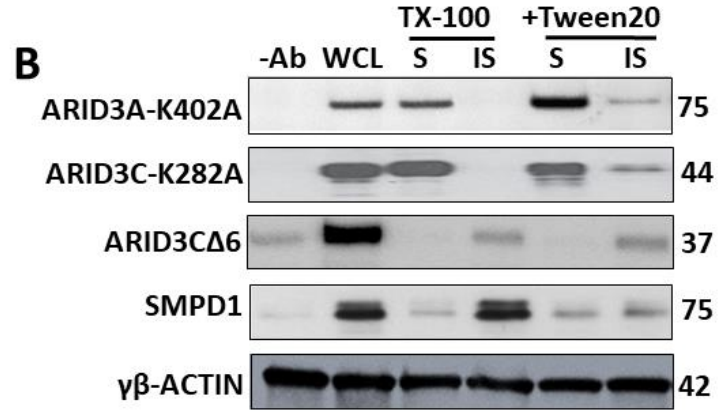

C

REKLES- $\beta$ point mutants

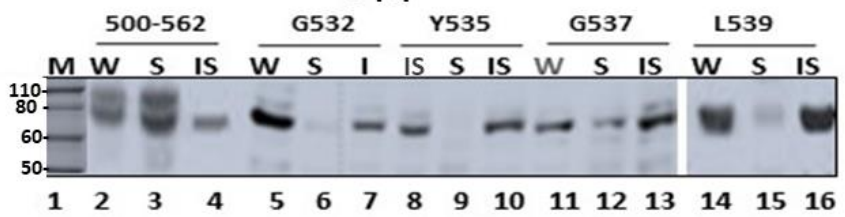

$\gamma \beta$-ACTIN 42

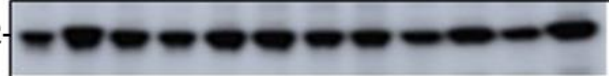

W: Whole Cell Lysate

S: Triton X-soluble

IS: Triton X-insoluble 


\section{Figure 4}

\begin{tabular}{|c|c|c|c|}
\hline $\mathbf{A}$ & & & spacer \\
\hline $1-601$ & Acidiç & ARID & REKLES \\
\hline d453-500 & & & \\
\hline d521-541 & & & 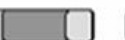 \\
\hline $1-541$ & & & \\
\hline d500-521 & & & \\
\hline
\end{tabular}

B
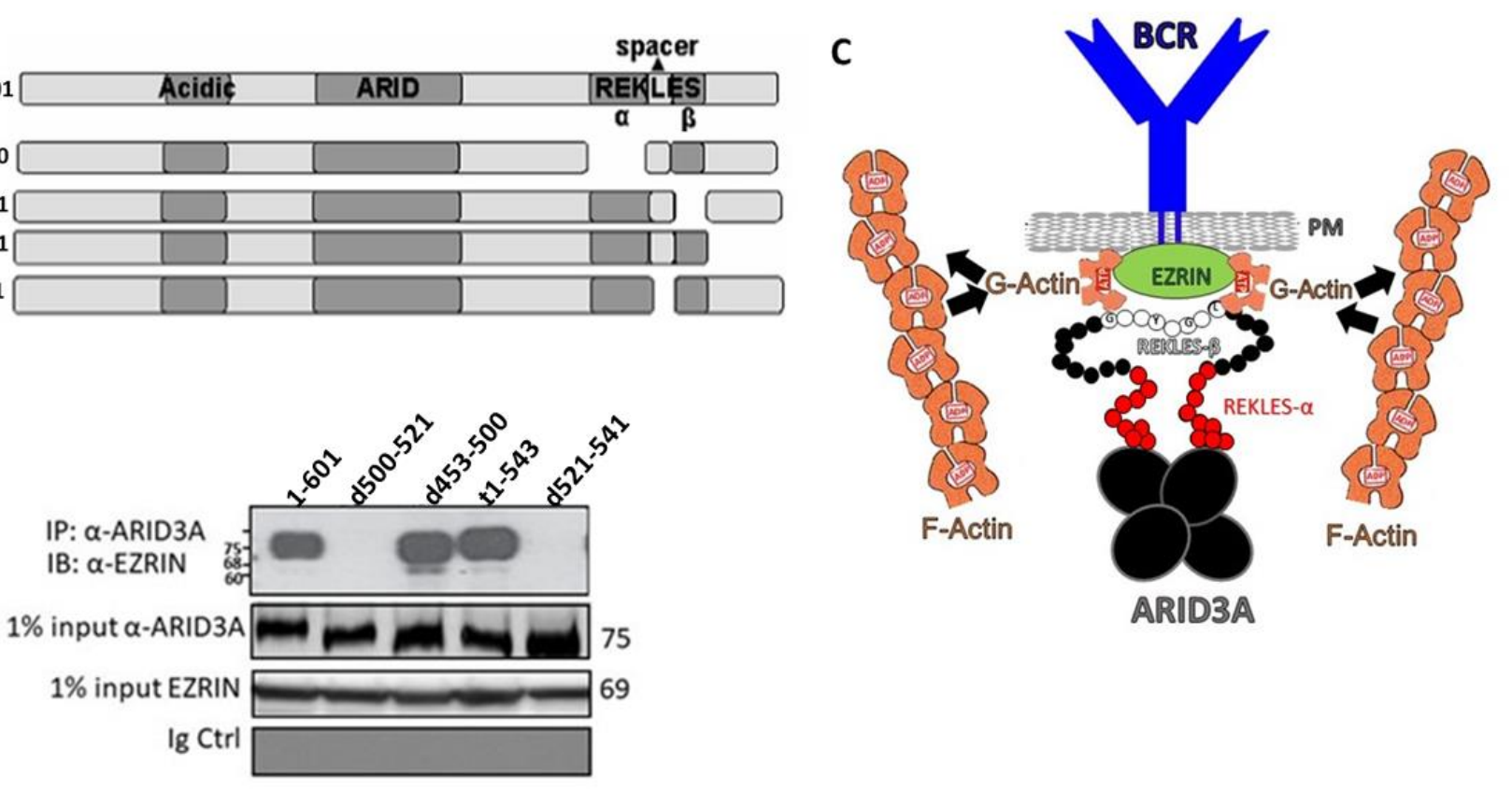

\section{S-Figure 1}

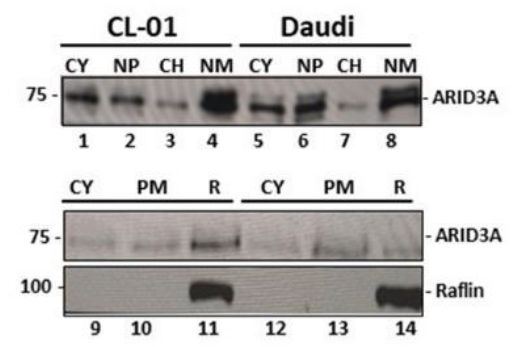

\section{S-Figure 2}

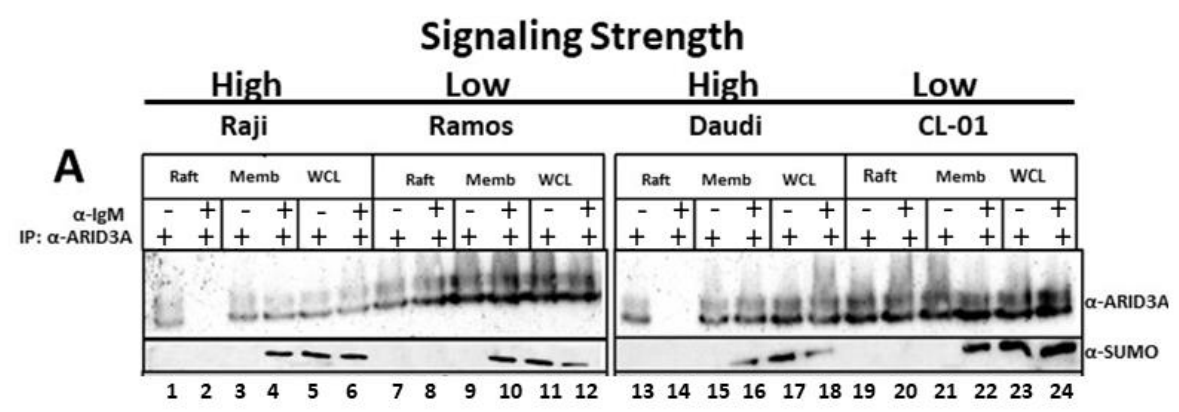




\section{S-Figure 3}

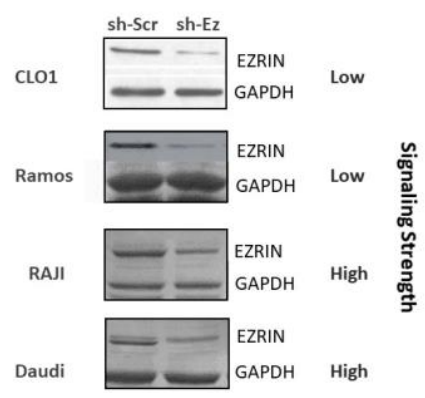

\section{S-Figure 4}

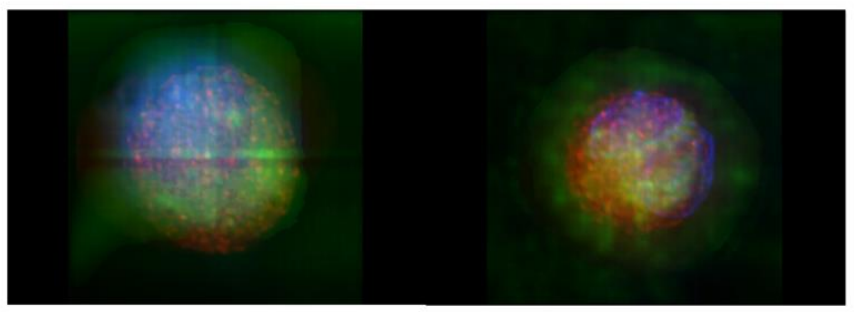

ARID3A

ACTIN

DNA

\section{S-Figure 5}

A

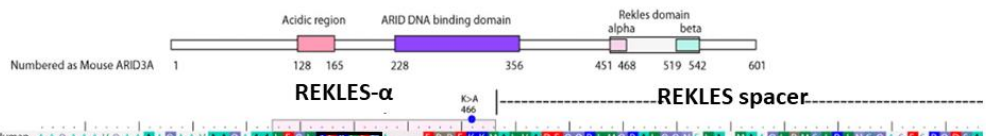

B
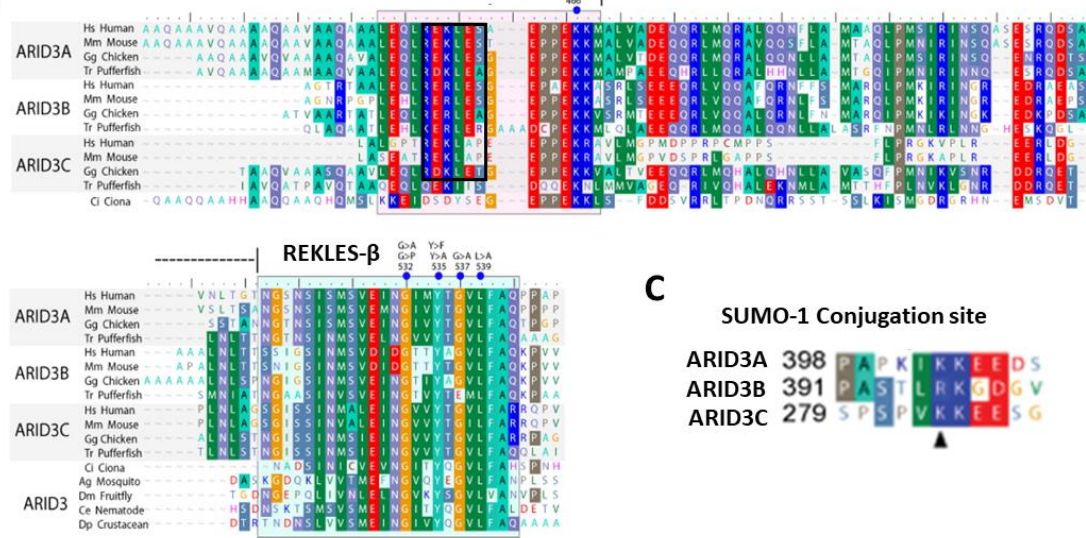

C

SUMO-1 Conjugation site

ARID3A 398

ARID3B 391

ARID3C 279 S P S

\section{S-Figure 6}

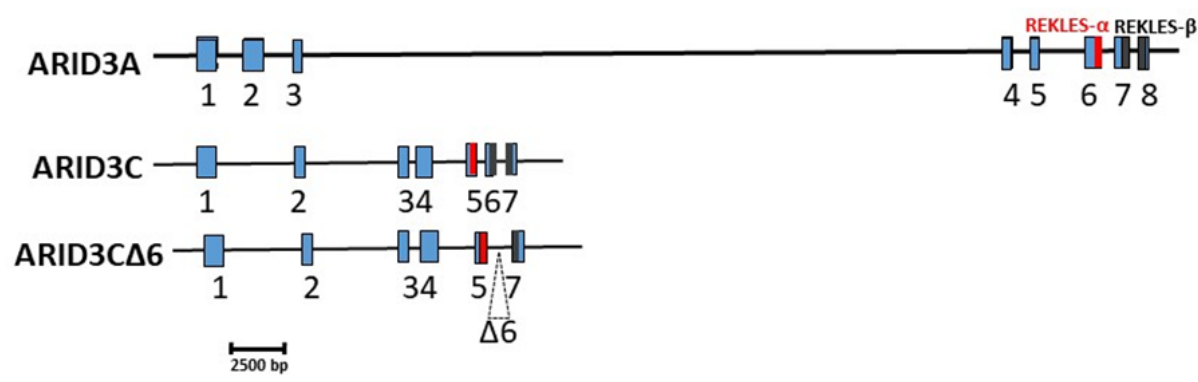

